\title{
Incidental Statin Use and the Risk of Stroke or Transient Ischemic Attack after Radiotherapy for Head and Neck Cancer
}

\author{
Daniel Addison, ${ }^{\mathrm{a}, \mathrm{b}, \mathrm{c}, \mathrm{d}}$ Patrick R. Lawler, ${ }^{\mathrm{e}, \mathrm{f}}$ Hamed Emami, ${ }^{\mathrm{a}, \mathrm{c}}$ Sumbal A. Janjua, ${ }^{\mathrm{a}}$ Pedro V. Staziaki, ${ }^{\mathrm{a}}$ \\ Travis R. Hallett, ${ }^{\mathrm{a}}$ Orla Hennessy, ${ }^{\mathrm{a}}$ Hang Lee, ${ }^{\mathrm{g}}$ Bálint Szilveszter, ${ }^{\mathrm{a}}$ Michael Lu, ${ }^{\mathrm{a}}$ Negar Mousavi, ${ }^{\mathrm{f}}$ \\ Matthew G. Nayor, ${ }^{\mathrm{f}}$ Francesca N. Delling, ${ }^{\mathrm{h}}$ Javier M. Romero, ${ }^{\mathrm{i}}$ Lori J. Wirth, ${ }^{\mathrm{j}}$ Annie W. Chan, ${ }^{\mathrm{k}}$ \\ Udo Hoffmann, ${ }^{\text {a }}$ Tomas G. Neilan ${ }^{\mathrm{a}, \mathrm{b}, \mathrm{c}}$ \\ ${ }^{a}$ Cardiac MR PET CT Program, Department of Radiology, Massachusetts General Hospital, Harvard Medical School, Boston, MA, USA \\ ${ }^{b}$ Cardio-Oncology Program, Division of Cardiology, Department of Medicine, Massachusetts General Hospital, Harvard Medical School, Boston, MA, USA \\ 'Division of Cardiology, Department of Medicine, Massachusetts General Hospital, Harvard Medical School, Boston, MA, USA \\ ${ }^{\mathrm{d} D i v i s i o n}$ of Cardiology, Department of Medicine, The Ohio State University, Columbus, OH, USA \\ ePeter Munk Cardiac Centre, Toronto General Hospital, and the Heart and Stroke Richard Lewar Centre of Excellence, University of Toronto, \\ Toronto, ON, Canada \\ ${ }^{f}$ Division of Cardiology, Department of Medicine, Brigham and Women's Hospital, Harvard Medical School, Boston, MA, USA \\ ${ }^{9}$ Biostatistics Center, Department of Medicine, Massachusetts General Hospital, Harvard Medical School, Boston, MA, USA \\ hDivision of Cardiology, Department of Medicine, University of California San Francisco, San Francisco, CA, USA \\ 'Division of Neuroradiology, Department of Radiology, Massachusetts General Hospital, Harvard Medical School, Boston, MA, USA \\ 'Division of Oncology, Department of Medicine, Massachusetts General Hospital, Harvard Medical School, Boston, MA, USA \\ ${ }^{k}$ Department of Radiation Oncology, Massachusetts General Hospital, Harvard Medical School, Boston, MA, USA
}

Background and Purpose Interventions to reduce the risk for cerebrovascular events (CVE; stroke and transient ischemic attack [TIA]) after radiotherapy (RT) for head and neck cancer (HNCA) are needed. Among broad populations, statins reduce CVEs; however, whether statins reduce CVEs after RT for HNCA is unclear. Therefore, we aimed to test whether incidental statin use at the time of RT is associated with a lower rate of CVEs after RT for HNCA.

Methods From an institutional database we identified all consecutive subjects treated with neck RT from 2002 to 2012 for HNCA. Data collection and event adjudication was performed by blinded teams. The primary outcome was a composite of ischemic stroke and TIA. The secondary outcome was ischemic stroke. The association between statin use and events was determined using Cox proportional hazard models after adjustment for traditional and RT-specific risk factors.

Results The final cohort consisted of 1,011 patients ( $59 \pm 13$ years, 30\% female, 44\% hypertension) with $288(28 \%)$ on statins. Over a median follow-up of 3.4 years (interquartile range, 0.1 to 14 ) there were 102 CVEs (89 ischemic strokes and 13 TIAs) with 17 in statin users versus 85 in nonstatins users. In a multivariable model containing known predictors of CVE, statins were associated with a reduction in the combination of stroke and TIA (hazard ratio [HR], 0.4; 95\% confidence interval $[\mathrm{Cl}$, 0.2 to $0.8 ; P=0.01$ ) and ischemic stroke alone ( $\mathrm{HR}, 0.4 ; 95 \% \mathrm{Cl}, 0.2$ to $0.8 ; P=0.01)$.

Conclusions Incidental statin use at the time of RT for HNCA is associated with a lower risk of stroke or TIA.
Correspondence: Tomas G. Neilan Cardiac MR PET CT Program, Department of Radiology and CardioOncology Program, Division of Cardiology, Department of Medicine, Massachusetts General Hospital, Havard Medical School, 165 Cambridge St, Boston, MA 02114, USA Tel: +1-617-724-5351

Fax: +1-617-726-6808 E-mail: tneilan@mgh.harvard.edu

Received: November 8, 2017 Revised: December 1, 2017 Accepted: December 5, 2017 


\section{Introduction}

Worldwide over 550,000 individuals are diagnosed with head and neck cancer each year, making it the sixth leading type of cancer. ${ }^{1}$ Radiotherapy (RT) is a standard primary treatment for most patients with head and neck cancer. ${ }^{2-6}$ With advancements in diagnosis and care, the 5-year survival for head and neck cancers has improved to over $65 \% .{ }^{7,8}$ With improved survival there has been an increased focus on the adverse longterm complications associated with successful cancer therapy. Consistent data have established an association between cancer therapies and an increased risk of cardiovascular disease and there is a 5- to 17-fold greater risk of cerebrovascular events (CVEs; stroke or transient ischemic attack [TIA]) in patients with head and neck cancer who are treated with RT compared to matched non-RT treated controls. ${ }^{9-11}$

The mechanisms for this increase in CVEs after RT that involves the neck are not completely understood, but available data suggest a role for direct injury to the carotid arteries causing increased inflammation, vascular dysfunction and accelerated atherosclerosis. ${ }^{10-14}$ Currently, no therapies have been shown to reduce the risk of CVEs after RT that involves the neck. In clinical trials among broad groups of patients with traditional risk factors for stroke, statins, as compared to placebo, have been associated with a $27 \%$ reduction in CVEs. ${ }^{15}$ The mechanisms behind the protective effect of statins against CVEs are thought to be related to a reduction in inflammation leading to stabilization or reversal in atherosclerotic plaque burden. ${ }^{16-20}$ Interestingly, these processes significantly overlap with key elements in the pathogenesis of radiation-induced carotid disease: inflammation and accelerated atherosclerosis. ${ }^{12-14}$ There is also scientific plausibility and preliminary clinical data to support the hypothesis that statins may reduce the risk for CVEs after RT that involves the neck. Specifically, in vitro data examining the impact of pravastatin on radiation treated endothelial cells showed a reduction in inflammation. ${ }^{21}$ In supportive clinical data, statins were associated a reduction in re-stenosis after carotid stenting in patients who developed symptomatic carotid stenosis after RT involving the neck. ${ }^{22}$ However, despite plausibility and supportive in vitro data, there are no clinical data testing the effects of statin therapy at the time of RT on the risk of CVEs among this vulnerable cohort. ${ }^{23,24}$ Therefore, we sought to test whether incidental statin use among head and neck cancer patients at the time of neck RT was associated with a decreased risk of CVEs. We hypothesize that statin use at the time of neck RT is associated with a decrease CVEs, even after accounting for expected reductions based on traditional cardiovascular risk factors.

\section{Methods}

\section{Study patients}

From an institutional database, we retrospectively identified all consecutive patients with head and neck cancer that underwent RT over an 11-year period from January 2002 to December 2012. Data collection, including cardiovascular and cancer-specific variables, and event adjudication was performed manually by two teams of independent investigators. Covariates of interest included age, gender, body mass index, baseline stroke prevention medications, history of hypertension, dyslipidemia, diabetes mellitus, chronic kidney disease, smoking, prior cerebrovascular disease (stroke or TIA), coronary artery disease (CAD), congestive heart failure and atrial fibrillation as well as serum mean low density lipoprotein (LDL) level at the time of RT, as previously defined. ${ }^{25,26}$ Cancer specific variables including cancer stage, surgical treatment, specified RT dose, chemotherapy use, and type of head and neck cancer were obtained from the electronic medical record. Cancer stage was categorized into localized/regional (stage I-III) and advanced/distant (stage IV) to account for the influence of median cancer-associated life-expectancy on the treatment decision and subsequent RT-associated events. Variables collected represented data available at the time of initiation of RT and were derived from the electronic medical record. The date of initial RT treatment was considered the study entry date. Specifically, statin use was defined based on the electronic medical record as documented at the time of initiation of RT. Continued statin use was assessed by review of the electronic records at 12 months post-RT initiation of 20 randomly selected baseline statin and non-statin users, respectively. We excluded patients who received neck RT prior to 2002 due to inconsistent data availability. We also excluded those with head and cancers who did not receive "neck" RT and those patients with incomplete RT records. The Human Subjects Research Review Committee of the Massachusetts General Hospital/Partners HealthCare approved the study protocol (Institutional Review Board \#2014P001394); given the retrospective nature of the study, informed consent was required.

\section{Outcomes}

The primary outcome of interest was the occurrence of a CVE following RT. This was defined as a composite of ischemic stroke and TIA. The primary outcome was chosen due to consistent data showing an increase in the combination of stroke and TIA post-RT for head and neck cancer. ${ }^{11,27-30}$ The secondary outcome was ischemic stroke alone. Events were ascertained via electronic medical record review and adjudicated by board certified investigators (blinded to statin use) using recom- 
mended standardized definitions for stroke and TIA from published consensus stroke definitions. ${ }^{31,32}$ Specifically, ischemic stroke was defined as an episode of neurological dysfunction caused by focal cerebral, spinal, or retinal infarction. Available computed tomography and magnetic resonance imaging reports were also reviewed for additional confirmation. TIA was defined as transient ( $<24$ hours) stroke symptoms and the absence of objective cerebral, spinal, or retinal infarction as seen with pathology or imaging.

\section{Statistical analysis}

Continuous data are presented as mean \pm standard deviation. Comparisons between groups (statin vs. non-statin users) were performed with the use of an independent sample t-test for continuous variables, Fisher exact test for categorical variables, and the Wilcox rank-sum test for ordinal variables. Hazard ratios (HR) for the association of statin use with events were estimated using Cox proportional hazard models with follow-up time used as the time scale. The assumption of proportionality was examined using Schoenfeld residuals and the interaction terms with time for each exposure variable and covariate. No substantial deviations from proportionality were observed. There were no missing covariates. Important covariates for CVEs were selected based on prior knowledge and were adjusted in multivariable analyses. These variables included known general and cancer-specific predictors of cerebrovascular disease, including age, gender, history of hypertension, dyslipidemia, diabetes mellitus, smoking, prior cerebrovascular disease (stroke or TIA), and CAD as well as RT dose. ${ }^{5,31,32}$ Event curves for both CVEs and ischemic stroke were determined according to the Kaplan-Meier method and comparisons were performed by the log-rank test. Cox proportional hazards model HR function curves comparing the statin group with the non-statin group were also constructed, accounting for significant covariables and competing risk. Censoring criteria included death from any cause (including cancer) or last documented visit for those without events or death. Additional exploratory analyses stratified in subgroups (median RT dose, individual stroke prevention medications, and those without distant/advanced disease) were also performed. We did not specifically focus on neck dissection as has not been clearly shown to correlate with stroke outcome following neck RT. ${ }^{33}$ In addition, number-needed-to-treat (NNT) calculations were performed to better assess the clinical impacts of statin use in this population, by calculation of the inverse of the absolute risk reduction(s). For all analyses a two-tailed $P$-value of $<0.05$ was considered significant. Statistical tests were performed using SAS version 9.4 (SAS institute, Cary, NC, USA).

\section{Results}

The final cohort included 1,011 patients with a median followup time from neck RT of 3.4 years (interquartile range, 1.4 to 6.0) (Figure 1). The mean age of the entire cohort at the time or RT was $59 \pm 13$ years (range, 10 to 89 ) and $30 \%$ were women (Table 1). Oropharyngeal carcinoma was the most common cancer type (Table 1). At the time of diagnosis, 21\% of patients had advanced or distant disease. From the cohort, 7\% were treated with RT alone, $82 \%$ treated with chemotherapy plus $\mathrm{RT}$, and $41 \%$ co-managed with surgery in addition to RT. Mean neck RT dose was $66.6 \pm 21.1 \mathrm{~Gy}$ (median 70.0; range, 4.0 to 196.0). The entire cohort was stratified according to the use of statins (Table 1); statin users were more likely to be older, hypertensive and have diabetes. There was no difference in cancer management strategies between statin users and non-users. Among statin users $>80 \%$ remained on therapy at 1 year.

Among the entire cohort, there were 102 CVEs over the median follow-up period of 3.4 years (range, 0.1 to 14 ). Over median follow-up periods of 3.2 and 3.5 years, respectively, there were 17 CVEs (16 strokes and one TIA) among incidental statin users compared to 85 events in the non-statin users (73 strokes and 12 TIAs), respectively. The median time to CVE was 3.0 years among statin users and 3.3 years among non-statin users. Figure 2 shows Kaplan-Meier CVE-free survival curves following RT therapy, stratified by statin use. Separation in the event curves became apparent after approximately 2.5 years and persisted through the duration of follow-up. In a multi-variable model, that included the traditional and RT-specific risk factors for CVEs, statin use at the time of RT was associated with a nearly $60 \%$ relative risk reduction in the risk of subsequent CVEs (adjusted Cox proportional HR of $0.41 ; 95 \%$ confidence interval [Cl], 0.21 to 0.79: $P=0.007$ ) (Table 2 and Figure 3). The resulting NNT to prevent any CVE during the median follow-up was 18.

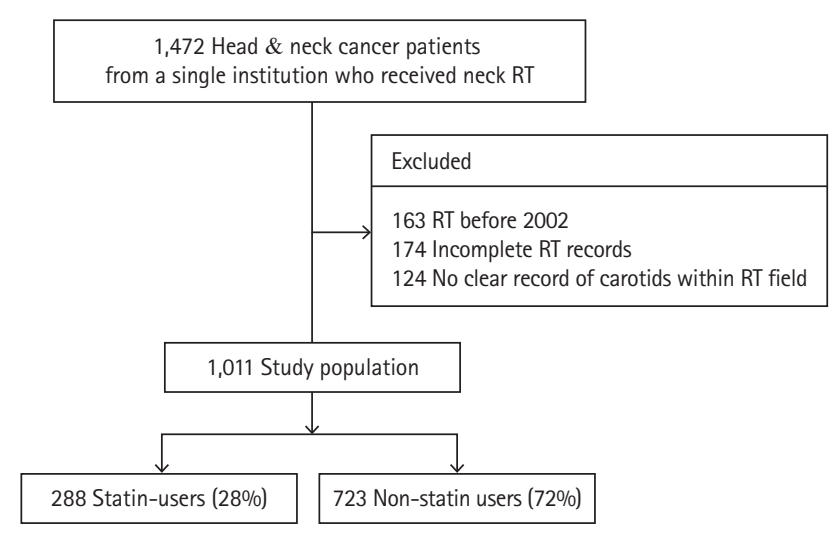

Figure 1. The study cohort. RT, radiotherapy. 
Table 1. Baseline characteristics by statin use

\begin{tabular}{|c|c|c|c|c|}
\hline Variable & $\begin{array}{l}\text { Overall cohort } \\
\quad(n=1,011)\end{array}$ & $\begin{array}{l}\text { Statin use } \\
(n=288)\end{array}$ & $\begin{array}{c}\text { Non-statin use } \\
(n=723)\end{array}$ & $P$ \\
\hline \multicolumn{5}{|l|}{ Demographic } \\
\hline Age (yr) & $59.4 \pm 13.4$ & $65.4 \pm 10.1$ & $57.0 \pm 13.8$ & $<0.001$ \\
\hline Female sex & $304(30.1)$ & 66 (22.9) & $238(32.9)$ & 0.002 \\
\hline BMI $\left(\mathrm{kg} / \mathrm{m}^{2}\right)$ & $27.5 \pm 5.7$ & $28.9 \pm 5.7$ & $26.5 \pm 5.6$ & 0.010 \\
\hline \multicolumn{5}{|l|}{ Traditional CVD risk factors } \\
\hline Hypertension & $447(44.2)$ & $198(68.7)$ & $249(34.4)$ & $<0.001$ \\
\hline Diabetes & $120(11.9)$ & $74(25.7)$ & $46(6.4)$ & $<0.001$ \\
\hline Dyslipidemia & $278(27.5)$ & $211(73.3)$ & $67(9.3)$ & $<0.001$ \\
\hline Mean LDL & $103.1 \pm 34.9$ & $95.7 \pm 33.2$ & $106.1 \pm 35.1$ & $<0.001$ \\
\hline Total cholesterol & $157.2 \pm 40.9$ & $153.2 \pm 36.7$ & $158.8 \pm 42.4$ & 0.057 \\
\hline Smoking history* & $665(65.8)$ & $206(71.5)$ & $459(63.5)$ & 0.019 \\
\hline Active smoking & $214(21.2)$ & $53(18.4)$ & $161(22.3)$ & 0.201 \\
\hline Carotid artery disease & $24(2.4)$ & $19(6.6)$ & $5(0.7)$ & $<0.001$ \\
\hline Coronary artery disease & $98(9.7)$ & $69(24.0)$ & $29(4.0)$ & 0.008 \\
\hline Prior CVE & $70(6.9)$ & 40 (13.9) & $30(4.2)$ & $<0.001$ \\
\hline Prior $\mathrm{CHF}$ & $41(4.1)$ & $24(8.3)$ & $17(2.4)$ & $<0.001$ \\
\hline Chronic kidney disease & $25(2.5)$ & $16(5.6)$ & $9(1.2)$ & $<0.001$ \\
\hline Atrial fibrillation & $42(4.2)$ & $17(5.9)$ & $25(3.5)$ & 0.083 \\
\hline Mean ASCVD 10-year risk (\%) & $10.1 \pm 11.5$ & $14.4 \pm 12.2$ & $8.9 \pm 11.0$ & $<0.001$ \\
\hline \multicolumn{5}{|l|}{ Radiation characteristics } \\
\hline Mean radiation dose (mSv) & $66.6 \pm 21.1$ & $66.4 \pm 18.2$ & $66.7 \pm 22.1$ & 0.130 \\
\hline Proton RT & $69(6.8)$ & $15(5.2)$ & $54(7.5)$ & 0.216 \\
\hline \multicolumn{5}{|l|}{ Cancer management } \\
\hline Chemotherapy & $810(80.1)$ & $223(77.4)$ & $587(81.2)$ & 0.190 \\
\hline Neck surgery & $561(55.5)$ & $169(58.7)$ & $392(54.2)$ & 0.207 \\
\hline Neck dissection & $419(41.4)$ & $129(44.8)$ & $290(40.1)$ & 0.179 \\
\hline RT alone & $73(7.2)$ & $23(8.0)$ & $50(6.9)$ & 0.591 \\
\hline \multicolumn{5}{|l|}{ Type of head \& neck cancer } \\
\hline Nasopharyngeal & $79(7.8)$ & $12(4.2)$ & $67(9.3)$ & 0.006 \\
\hline Oropharyngeal & $470(46.5)$ & $131(45.5)$ & $339(47.0)$ & 0.676 \\
\hline Hypopharyngeal & $63(6.2)$ & $26(9.0)$ & $37(5.1)$ & 0.020 \\
\hline Laryngeal & $110(10.9)$ & $42(14.6)$ & $68(9.4)$ & 0.025 \\
\hline Other & $331(32.8)$ & 89 (30.9) & $242(33.5)$ & 0.061 \\
\hline Metastatic disease at presentation & $214(21.2)$ & $52(18.1)$ & $162(22.4)$ & 0.147 \\
\hline \multicolumn{5}{|l|}{ Medications } \\
\hline Aspirin & $248(24.6)$ & $140(48.6)$ & 108 (15.0) & $<0.001$ \\
\hline Clopidogrel & $18(1.8)$ & $14(4.9)$ & $4(0.6)$ & $<0.001$ \\
\hline Warfarin & $42(4.2)$ & $18(6.2)$ & $25(3.5)$ & 0.060 \\
\hline ACEi & $203(20.1)$ & 107 (37.2) & 96 (13.3) & $<0.001$ \\
\hline
\end{tabular}

Values are presented as mean $\pm \mathrm{SD}$ or number $(\%)$.

$\mathrm{BMI}$, body mass index; CVD, cerebrovascular disease; LDL, low density lipoprotein; CVE, cerebrovascular event; CHF, congestive heart failure; ASCVD, atherosclerotic cardiovascular disease; RT, radiation; ACEi, angiotensin-converting enzyme inhibitor.

*Smoking history: active or prior smoking. 
Table 2. Multivariable cox regression analysis for post-radiotherapy cerebrovascular event

\begin{tabular}{lccc}
\hline Variable & $\mathrm{HR}(95 \% \mathrm{Cl})$ & Chi-square & $P$ \\
\hline Statin use & $0.41(0.21-0.80)$ & 6.97 & 0.008 \\
Age & $1.04(1.02-1.06)$ & 22.84 & $<0.001$ \\
Male sex & $0.92(0.59-1.42)$ & 0.15 & 0.701 \\
Hypertension & $1.39(0.91-2.13)$ & 2.33 & 0.127 \\
Diabetes & $1.09(0.57-2.08)$ & 0.08 & 0.782 \\
Prior CAD & $1.07(0.50-2.26)$ & 0.03 & 0.868 \\
Prior CVE & $1.60(0.72-3.55)$ & 1.32 & 0.251 \\
Dyslipidemia & $0.73(0.39-1.37)$ & 0.96 & 0.326 \\
Smoking* & $1.19(0.90-1.58)$ & 1.47 & 0.226 \\
Radiation dose & $1.0(1.0)$ & 0.50 & 0.477 \\
\hline
\end{tabular}

$\mathrm{HR}$, hazard ratio; $\mathrm{Cl}$, confidence interval; $\mathrm{CAD}$, coronary artery disease; $\mathrm{CVE}$, cerebrovascular event. *Smoking: active or prior smoking.

Composite of ischemic stroke and TIA

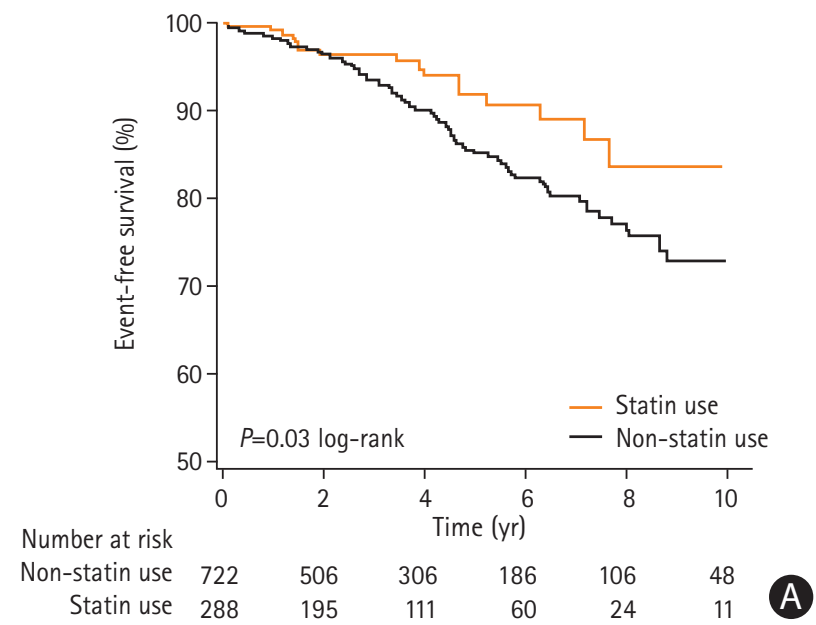

Ischemic stroke

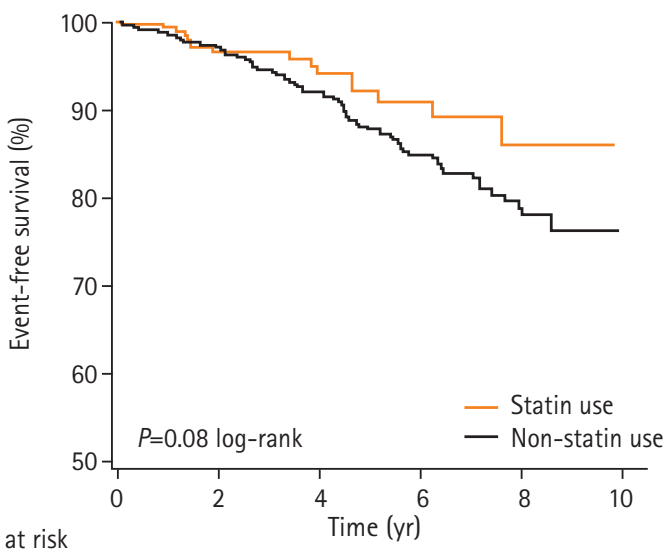

Number at risk

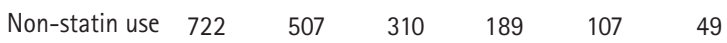

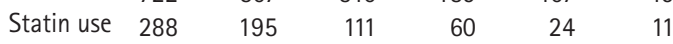

Figure 2. Post-neck radiotherapy cerebrovascular event-free survival (A) and post-neck radiotherapy ischemic stroke event-free survival (B), by incidental statin use. TIA, transient ischemic attack.

The secondary end-point was ischemic stroke. Over followup there were 89 ischemic strokes in the entire cohort, with 16 ischemic strokes among the statin-treated group and 73 in the non-statin group. Figure 2 also shows the Kaplan-Meier strokefree survival curves following neck RT, stratified by statin use. Separation in stroke-free survival curves became apparent within approximately 3 years and persisted through the duration of follow-up. In a similar multi-variable model, statin use was associated with a $>50 \%$ relative risk reduction in the development of post-RT ischemic stroke (adjusted $H R, 0.42 ; 95 \%$ $\mathrm{Cl}, 0.21$ to $0.83 ; P=0.01$ ) (Table 3 ). The resulting NNT to prevent a single ischemic stroke was 22 .

We tested the effect of statin use on both the primary and secondary endpoints stratified by median RT dose and the use of concurrent stroke prevention medications (Supplementary
Tables 1-4). There were 498 subjects with 54 CVEs in the lower median radiation dose group ( $<70 \mathrm{~Gy}$ ) and 513 subjects with 47 CVEs in the higher median radiation group. Following adjustment, statin use was associated with a HR of $0.36(P=0.02)$ among the $<70$ Gy RT group and a HR of $0.55(P=0.23)$ among those with higher dose of RT. We also tested the effect of concurrent antiplatelet (Aspirin and/or Plavix) and anti-thrombotic (Coumadin) use to the model. Following this, statin use remained associated with a decrease in events (adjusted HR, $0.41 ; 95 \% \mathrm{Cl}, 0.21$ to $0.79 ; P=0.01$ ). In addition, we performed a subgroup analysis among those without distant or advanced disease testing the effect of statins in those with longer lifeexpectancies. Among those without advanced disease, statin use was still associated with a decrease in events (adjusted HR, $0.40 ; 95 \% \mathrm{Cl}, 0.20$ to $0.79 ; P=0.008)$. Over the duration of fol- 
Table 3. Multivariable Cox regression analysis for post-radiotherapy ischemic stroke

\begin{tabular}{lccc}
\hline Variable & HR $(95 \% \mathrm{Cl})$ & Chi-square & $P$ \\
\hline Statin use & $0.42(0.21-0.83)$ & 6.18 & 0.010 \\
Age & $1.04(1.03-1.06)$ & 22.36 & $<0.001$ \\
Male sex & $0.94(0.59-1.50)$ & 0.06 & 0.805 \\
Hypertension & $1.34(0.86-2.11)$ & 1.65 & 0.199 \\
Diabetes & $0.98(0.48-2.00)$ & $<0.01$ & 0.962 \\
Prior CAD & $1.23(0.57-2.63)$ & 0.27 & 0.602 \\
Prior CVE & $1.76(0.78-3.94)$ & 1.86 & 0.173 \\
Dyslipidemia & $0.79(0.41-1.53)$ & 0.48 & 0.487 \\
Smoking* & $1.28(0.95-1.74)$ & 2.60 & 0.107 \\
Radiation dose & $1.0(1.0)$ & 0.02 & 0.875 \\
\hline
\end{tabular}

$\mathrm{HR}$, hazard ratio; $\mathrm{Cl}$, confidence interval; $\mathrm{CAD}$, coronary artery disease; $\mathrm{CVE}$, cerebrovascular event.

*Smoking: active or prior smoking.

low-up (median, 3.4 years), there were 317 deaths (41\%). There was no difference in all-cause mortality according to statin status (92 [40\%] vs. $225[41 \%], P=0.75)$.

\section{Discussion}

We performed a retrospective study testing the effect of incidental statin use on the risk of stroke and TIA after RT that involved the neck. In this study of over 1,000 patients with head and neck cancer treated with RT, incidental statin use at the time of RT was associated with a reduction in the risk for subsequent CVEs. Of the patients treated with statin therapy at the time of their RT, those on statins saw a nearly $60 \%$ reduction in the combined primary endpoint of ischemic stroke and TIA and a $>50 \%$ relative risk reduction in the development of ischemic stroke. These reductions remained, even after adjustment for stroke risk factors, radiation dose, and exclusion of persons with advanced cancer disease, despite the higher traditional atherosclerotic cardiovascular disease risk profile noted among statin users in this cohort. During of the study followup period (median, 3.4 years), the NNT to prevent any CVE was 18 , and the NNT to prevent one ischemic stroke was 22. By comparison, the estimated NNT with statins for the primary prevention of stroke among persons $>65$ years was estimated at 142 and 196 in the overall general adult population, suggesting enhanced effect following RT. ${ }^{34,35}$ To our knowledge, these are the first data demonstrating an associated reduction in ischemic stroke and TIA with statin therapy among a broad population of RT-treated patients.

The effect of statin therapy on the risk of CVEs not related to RT was evaluated by the Stroke Prevention by Aggressive Reduction in Cholesterol Levels (SPARCL) Investigators. ${ }^{36}$ In a co- hort of over 4,700 patients, the use of high-dose atorvastatin was associated with significant reductions in stroke and TIA $(23 \%)$. At the time of publication, the investigators suggested that the primary reason for the reduction was the decrease in LDL levels. However, the authors also considered the potential role of non-lipid lower effects, such as a reduction in inflammation, of statins on the long-term risk of stroke. These "pleitropic" effects of statins are supported by the findings of the primary stroke prevention trial, Anglo-Scandinavian Cardiac Outcomes Trial- Lipid Lowering Arm (ASCOT-LLA). ${ }^{15}$ In this trial, moderate intensity statin therapy provided a nearly 30\% reduction in the risk of stroke, an affect that appears disproportionate to the reduction in LDL. This in concert with other available prevention data helped lead to the incorporation of statins into the stroke prevention algorithm in those at elevated stroke-risk, irrespective of baseline LDL. ${ }^{37,38}$ However, there are no data testing rationale interventions to reduce CVEs in patients after RT to the neck. In study, the observed CVE reductions were well over 50\%, a number in far excess to the magnitude of reduction seen in traditional non-irradiated populations. While retrospective, this is the first analysis examining the clinical impact of statins on the risk of CVEs following neck $\mathrm{RT}$ and the first to define a potential rational intervention for this population, particularly given the disproportionate magnitude of CVE reduction observed beyond that expected based on traditional risk factors alone. ${ }^{38}$

These results are supported by other mechanistic data. Seminal animal studies from nearly five decades ago described a link between dyslipidemia and the development of radiation-induced carotid artery stenosis. ${ }^{39-41}$ Subsequent human studies have since validated these associations. ${ }^{13}$ Statins are known to modulate the risk of vascular events in patients with underlying 


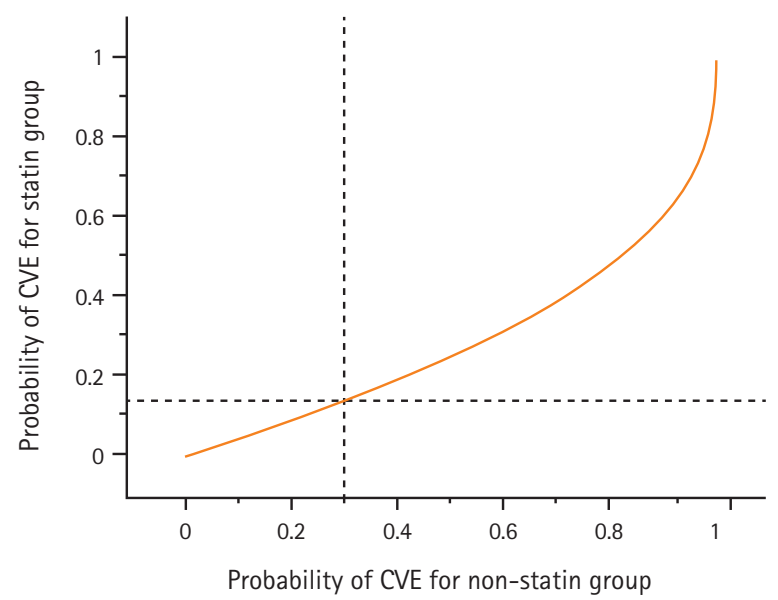

Figure 3. Relationship between probabilities of a cerebrovascular event (CVE) for statin and non-statin users under the Cox regression model. Survival functions of study subjects to waiting times for a CVE event between the two groups are related as $\mathrm{S} 1(\mathrm{t})=[\mathrm{SO}(\mathrm{t})] \mathrm{c}$ (statin yes $=1 ; n 0=0)$, where $\mathrm{t}$, is time of follow-up and c, is the estimated hazard ratio ( 0.41 for the statin use for events). 1-SO(t) represents the horizontal axis and corresponding $1-\mathrm{S} 1(\mathrm{t})$ the vertical axis. The dashed lines indicate that if the probability of observing at least 1 event by time $t$ (e.x., 0.30 for the no statin group, the corresponding probability for the statin group is 0.14 ).

dyslipidemia. Yet, statins also attenuate this risk in populations without markedly elevated lipid levels, but with otherwise elevated risk and vascular dysfunction like diabetics. ${ }^{42}$ Moreover, Paraskevas et al. ${ }^{43}$ postulated that irradiated carotid arteries may be more susceptible to elevations in cholesterol irrespective of baseline LDL. RT itself has been observed to alter the vasculature by the induction of endothelial dysfunction, increased inflammation, oxidative stress, and progressive capillary luminal fibrin and platelet-mediated destruction leading to potential downstream events. However, Gaugler et al..$^{21}$ demonstrated a downregulation of vascular endothelial dysfunction (transcription factor activator protein 1, monocyte chemoattractant protein 1, interleukin 6 [IL-6], and IL-8), inflammation (activator protein 1) and thrombosis (decreased leukocyte and platelet adhesion) through statin treatment following RT exposure. In addition, statins have also been associated with reductions in oxidative stress. ${ }^{44}$ Since RT acts to modulate vascular architecture and accelerate atherosclerosis using pathways affected by statin therapy, it may be reasonable to postulate that statins themselves may serve to attenuate the vascular disruptions induced by RT, allowing for enhanced reductions in observed clinical events beyond rates seen in non-irradiated populations and estimated based on traditional factors alone. ${ }^{19-21,43-45}$ However, further studies are needed.

Our study has several limitations. Given that the subjects studied were enrolled at a tertiary care center where patients received treatment, but not necessarily long-term follow-up, it was difficult to ascertain the duration of statin therapy. Statin use was recorded at the time of RT and the duration of use (or non-use) was up to the primary team. Yet, among those sampled statin use or non-use remained high, even at 1 year follow-up. Additionally, due to practice variations across the duration of the study, we did not focus on statin intensity or class of agent used. Yet, considering the significance of the reduction observed, even after adjusting for LDL, it is reasonable to suspect that the benefits of statin therapy in this population extend well beyond the reductions in LDL and therefore are less likely to be limited to statin class or intensity. Misclassification of stroke was highly unlikely because the clinical symptoms were correlated with neuroimaging. This cross-referencing of events also helped to minimize recall bias. However, TIAs may have been misclassified as seizures or migraines, and therefore undercounted, despite physician adjudication. This is an unavoidable problem when using TIA as an outcome, and the separate analysis evaluating only stroke was performed to obviate this possible bias. We considered the use of propensitymatching to adjust for potential confounders. However, in view of an adequately-powered event rate relative to the number of cofounders, conventional regression was used for the analyses. Finally, we did not note any major hemorrhagic strokes in our cohort, a finding that may at least in-part be explained by the relative paucity of aggressive anti-platelet and potent anticoagulant regimens noted in this population.

\section{Conclusions}

Survivors of head and neck cancer treated with neck RT are at high-risk for future CVEs, including ischemic stroke and TIA. Statin use at the time of RT appears to be associated with a reduction of that risk, even after accounting for expected reductions based on more traditional risk factors. Future prospective studies examining the role of statins in head and neck cancer patients who receive RT, without traditional indications for statins are needed.

\section{Supplementary materials}

Supplementary materials related to this article can be found online at https://doi.org/10.5853/jos.2017.01802.

\section{Disclosure}

The authors have no financial conflicts of interest. 


\section{Acknowledgments}

Tomas G. Neilan has the following support: The Kohlberg Foundation, an American Heart Association Fellow to Faculty Award (12FTF12060588), National Institutes of Health/ National Heart, Lung, and Blood Institute (1R01HL130539-01A1; 1R01HL137562-01A1) and National Institutes of Health/Harvard Center for AIDS Research (P30 Al060354). Daniel Addison and Sumbal A. Janjua were supported by NIH/NHLBI 5T32HL076136.

\section{References}

1. Jemal A, Bray F, Center MM, Ferlay J, Ward E, Forman D. Global cancer statistics. CA Cancer J Clin 2011;61:69-90.

2. Fletcher GH, Evers WT. Radiotherapeutic management of surgical recurrences and postoperative residuals in tumors of the head and neck. Radiology 1970;95:185-188.

3. Snow GB, Annyas AA, van Slooten EA, Bartelink $H$, Hart AA. Prognostic factors of neck node metastasis. Clin Otolaryngol Allied Sci 1982;7:185-192.

4. Kramer S, Gelber RD, Snow JB, Marcial VA, Lowry LD, Davis LW, et al. Combined radiation therapy and surgery in the management of advanced head and neck cancer: final report of study 73-03 of the Radiation Therapy Oncology Group. Head Neck Surg 1987;10:19-30.

5. Bernier J, Domenge C, Ozsahin M, Matuszewska K, Lefèbvre $\mathrm{JL}$, Greiner $\mathrm{RH}$, et al. Postoperative irradiation with or without concomitant chemotherapy for locally advanced head and neck cancer. N Engl J Med 2004;350:1945-1952.

6. Haddad RI, Shin DM. Recent advances in head and neck cancer. N Engl J Med 2008;359:1143-1154.

7. National Cancer Institute. Cancer trends progress report: 2011/2012 update. https://progressreport.cancer.gov/sites/default/ files/archive/report2011.pdf. 2014. Accessed January 2, 2018.

8. Bonner JA, Harari PM, Giralt J, Azarnia N, Shin DM, Cohen RB, et al. Radiotherapy plus cetuximab for squamous-cell carcinoma of the head and neck. N Engl J Med 2006;354:567-578.

9. Dorresteijn LD, Kappelle AC, Boogerd W, Klokman WJ, Balm $A J$, Keus RB, et al. Increased risk of ischemic stroke after radiotherapy on the neck in patients younger than 60 years. $J$ Clin Oncol 2002;20:282-288.

10. De Bruin ML, Dorresteijn $L D$, van't Veer MB, Krol AD, van der $\mathrm{Pal} H J$, Kappelle $A C$, et al. Increased risk of stroke and transient ischemic attack in 5-year survivors of Hodgkin lymphoma. J Natl Cancer Inst 2009;101:928-937.

11. Plummer C, Henderson RD, O'Sullivan JD, Read SJ. Ischemic stroke and transient ischemic attack after head and neck ra- diotherapy: a review. Stroke 2011;42:2410-2418.

12. Zidar N, Ferluga D, Hvala A, Popović M, Soba E. Contribution to the pathogenesis of radiation-induced injury to large arteries. J Laryngol Otol 1997;111:988-990.

13. Silverberg GD, Britt RH, Goffinet DR. Radiation-induced carotid artery disease. Cancer 1978;41:130-137.

14. Gujral DM, Chahal N, Senior R, Harrington KJ, Nutting CM. Radiation-induced carotid artery atherosclerosis. Radiother Oncol 2014;110:31-38.

15. Sever PS, Dahlöf B, Poulter NR, Wedel H, Beevers $G$, Caulfield $M$, et al. Prevention of coronary and stroke events with atorvastatin in hypertensive patients who have average or lowerthan-average cholesterol concentrations, in the Anglo-Scandinavian Cardiac Outcomes Trial: Lipid Lowering Arm (ASCOT-LLA): a multicentre randomised controlled trial. Lancet 2003;361:1149-1158.

16. Albert MA, Danielson E, Rifai N, Ridker PM; PRINCE Investigators. Effect of statin therapy on C-reactive protein levels: the pravastatin inflammation/CRP evaluation (PRINCE): a randomized trial and cohort study. JAMA 2001;286:64-70.

17. Nissen $S E$, Tuzcu EM, Schoenhagen P, Brown BG, Ganz P, Vogel $R A$, et al. Effect of intensive compared with moderate lipidlowering therapy on progression of coronary atherosclerosis: a randomized controlled trial. JAMA 2004;291:1071-1080.

18. Ridker PM, Rifai N, Clearfield M, Downs JR, Weis SE, Miles $J S$, et al. Measurement of C-reactive protein for the targeting of statin therapy in the primary prevention of acute coronary events. N Eng/ J Med 2001;344:1959-1965.

19. Moreno PR, Kini A. Resolution of inflammation, statins, and plaque regression. JACC Cardiovasc Imaging 2012;5:178-181.

20. Antonopoulos AS, Margaritis M, Lee R, Channon K, Antoniades $C$. Statins as anti-inflammatory agents in atherogenesis: molecular mechanisms and lessons from the recent clinical trials. Curr Pharm Des 2012;18:1519-1530.

21. Gaugler MH, Vereycken-Holler V, Squiban C, Vandamme M, Vozenin-Brotons MC, Benderitter M. Pravastatin limits endothelial activation after irradiation and decreases the resulting inflammatory and thrombotic responses. Radiat Res 2005;163:479-487.

22. Favre JP, Nourissat A, Duprey A, Nourissat G, Albertini JN, Becquemin JP, et al. Endovascular treatment for carotid artery stenosis after neck irradiation. J Vasc Surg 2008;48:852-858.

23. Yusuf SW, Sami S, Daher IN. Radiation-induced heart disease: a clinical update. Cardiol Res Pract 2011;2011:317659.

24. Xu J, Cao Y. Radiation-induced carotid artery stenosis: a comprehensive review of the literature. Interv Neurol 2014;2:183-192.

25. Neilan TG, Mongeon FP, Shah RV, Coelho-Filho O, Abbasi SA, Dodson JA, et al. Myocardial extracellular volume expansion 
and the risk of recurrent atrial fibrillation after pulmonary vein isolation. JACC Cardiovasc Imaging 2014;7:1-11.

26. Neilan TG, Farhad H, Dodson JA, Shah RV, Abbasi SA, Bakker $J P$, et al. Effect of sleep apnea and continuous positive airway pressure on cardiac structure and recurrence of atrial fibrillation. J Am Heart Assoc 2013;2:e000421.

27. Wilbers J, Kappelle AC, Kessels RP, Steens SC, Meijer FJ, Kaanders $\mathrm{JH}$, et al. Long term cerebral and vascular complications after irradiation of the neck in head and neck cancer patients: a prospective cohort study: study rationale and protocol. BMC Neurol 2014;14:132.

28. Smith GL, Smith BD, Buchholz TA, Giordano SH, Garden AS, Woodward WA, et al. Cerebrovascular disease risk in older head and neck cancer patients after radiotherapy. J Clin Oncol 2008;26:5119-5125.

29. Swisher-McClure S, Mitra N, Lin A, Ahn P, Wan F, O'Malley B, et al. Risk of fatal cerebrovascular accidents after external beam radiation therapy for early-stage glottic laryngeal cancer. Head Neck 2014;36:611-616.

30. Hong JC, Kruser TJ, Gondi V, Mohindra P, Cannon DM, Harari $\mathrm{PM}$, et al. Risk of cerebrovascular events in elderly patients after radiation therapy versus surgery for early-stage glottic cancer. Int J Radiat Oncol Biol Phys 2013;87:290-296.

31. Easton JD, Saver JL, Albers GW, Alberts MJ, Chaturvedi S, Feld-

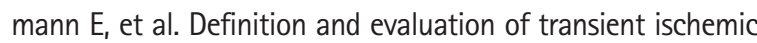
attack: a scientific statement for healthcare professionals from the American Heart Association/American Stroke Association Stroke Council; Council on Cardiovascular Surgery and Anesthesia; Council on Cardiovascular Radiology and Intervention; Council on Cardiovascular Nursing; and the Interdisciplinary Council on Peripheral Vascular Disease. The American Academy of Neurology affirms the value of this statement as an educational tool for neurologists. Stroke 2009;40:2276-2293.

32. Sacco RL, Kasner SE, Broderick JP, Caplan LR, Connors JJ, Culebras $A_{\text {, et }}$ al. An updated definition of stroke for the 21st century: a statement for healthcare professionals from the American Heart Association/American Stroke Association. Stroke 2013;44:2064-2089.

33. Chang CF, Kuo YL, Pu C, Chou YJ. Neck dissection and stroke in patients with oral cavity cancer: a population-based cohort study. Head Neck 2017;39:63-70.

34. Sigurdsson AF. Benefits of statins in healthy elderly subjects: what is the number needed to treat? JAm Coll Cardiol 2014;63:2302.

35. Taylor F, Huffman MD, Macedo AF, Moore TH, Burke M, Davey Smith $\mathrm{G}$, et al. Statins for the primary prevention of cardiovas- cular disease. Cochrane Database Syst Rev 2013;(1):CD004816.

36. Amarenco P, Bogousslavsky J, Callahan A 3rd, Goldstein LB,

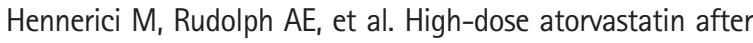
stroke or transient ischemic attack. N Engl J Med 2006;355: 549-559.

37. Meschia JF, Bushnell C, Boden-Albala B, Braun LT, Bravata DM, Chaturvedi $S$, et al. Guidelines for the primary prevention of stroke: a statement for healthcare professionals from the American Heart Association/American Stroke Association. Stroke 2014;45:3754-3832.

38. Stone NJ, Robinson JG, Lichtenstein AH, Bairey Merz CN, Blum $\mathrm{CB}$, Eckel RH, et al. American College of Cardiology/American Heart Association Task Force on Practice Guidelines. 2013 ACC/ AHA guideline on the treatment of blood cholesterol to reduce atherosclerotic cardiovascular risk in adults: a report of the American College of Cardiology/American Heart Association Task Force on Practice Guidelines. Circulation 2014;129(25 Suppl 2):S1-S45. Erratum in: Circulation 2014;129(25 Suppl 2):S46-S48.

39. Gold H. Production of arteriosclerosis in the rat. Effect of $x-$ ray and a high-fat diet. Arch Pathol 1961;71:268-273.

40. Vesselinovitch D, Wissler RW. Experimental production of atherosclerosis in mice. 2. Effects of atherogenic and highfat diets on vascular changes in chronically and acutely irradiated mice. J Atheroscler Res 1968;8:497-523.

41. Lamberts HB, de BW. Contributions to the study of immediate and early $x$-ray reactions with regard to chemo-protection. VII. X-ray-induced atheromatous lesions in the arterial wall of hypercholesterolaemic rabbits. Int J Radiat Biol Relat Stud Phys Chem Med 1963;6:343-350.

42. Cholesterol Treatment Trialists' (CTT) Collaborators, Kearney PM, Blackwell L, Collins R, Keech A, Simes J, et al. Efficacy of cholesterol-lowering therapy in 18,686 people with diabetes in 14 randomised trials of statins: a meta-analysis. Lancet 2008;371:117-125.

43. Paraskevas KI, Giannoukas AD, Mikhailidis DP. Irradiation-induced carotid artery stenosis: a preventable complication of neck radiotherapy? Angiology 2009;60:273-275.

44. Morawietz H, Erbs S, Holtz J, Schubert A, Krekler M, Goettsch $W_{1}$ et al. Endothelial protection, AT1 blockade and cholesterol-dependent oxidative stress: the EPAS trial. Circulation 2006;114(1 Suppl):I296-I301.

45. Weintraub NL, Jones WK, Manka D. Understanding radiationinduced vascular disease. J Am Coll Cardiol 2010;55:12371239. 
Supplementary Table 1. Risk of cerebrovascular events following neck radiotherapy using a multivariable Cox regression model for those treated with $<70 \mathrm{~Gy}$

\begin{tabular}{lccr}
\hline Variable & $\mathrm{HR}(95 \% \mathrm{Cl})$ & Chi-square & \multicolumn{1}{c}{$P$} \\
\hline Statin use & $0.39(0.15-1.02)$ & 3.66 & 0.056 \\
Age & $1.05(1.03-1.08)$ & 16.21 & $<0.001$ \\
Male sex & $1.06(0.54-2.08)$ & 0.03 & 0.855 \\
Hypertension & $0.99(0.52-1.92)$ & $<0.01$ & 0.987 \\
Diabetes & $0.91(0.31-2.65)$ & 0.03 & 0.862 \\
Prior CAD & $1.13(0.34-3.73)$ & 0.04 & 0.839 \\
Prior CVE & $1.55(0.51-4.73)$ & 0.60 & 0.438 \\
Dyslipidemia & $0.66(0.26-1.69)$ & 0.74 & 0.389 \\
Smoking* & $1.33(0.86-2.07)$ & 1.67 & 0.196 \\
\hline
\end{tabular}

$\mathrm{HR}$, hazard ratio; $\mathrm{Cl}$, confidence interval; $\mathrm{CAD}$, coronary artery disease; $C V E$, cerebrovascular event.

*Smoking: active or prior smoking. 
Supplementary Table 2. Risk of cerebrovascular events following neck radiotherapy using a multivariable Cox regression model for those treated with $\geq 70$ Gy

\begin{tabular}{lccc}
\hline Variable & HR $(95 \% \mathrm{Cl})$ & Chi-square & $P$ \\
\hline Statin use & $0.44(0.19-1.03)$ & 3.55 & 0.059 \\
Age & $1.03(1.01-1.06)$ & 6.18 & 0.013 \\
Male sex & $0.86(0.47-1.56)$ & 0.25 & 0.620 \\
Hypertension & $1.82(1.03-3.22)$ & 4.29 & 0.038 \\
Diabetes & $0.65(0.25-1.67)$ & 0.80 & 0.371 \\
Prior CAD & $0.90(0.34-2.38)$ & 0.05 & 0.828 \\
Prior CVE & $1.17(0.36-3.86)$ & 0.07 & 0.792 \\
Dyslipidemia & $0.80(0.36-1.79)$ & 0.28 & 0.803 \\
Smoking & $1.12(0.77-1.64)$ & 0.37 & 1.124 \\
\hline
\end{tabular}

$\mathrm{HR}$, hazard ratio; $\mathrm{Cl}$, confidence interval; $\mathrm{CAD}$, coronary artery disease; $\mathrm{CVE}$, cerebrovascular event.

*Smoking: active or prior smoking. 
Supplementary Table 3. Risk of cerebrovascular events following neck radiotherapy using a multivariable Cox regression model including other stroke reduction medications

\begin{tabular}{lccr}
\hline Variable & HR $(95 \%$ Cl) & Chi-square & 0.008 \\
\hline Statin use & $0.41(0.21-0.79)$ & 6.99 & $<0.001$ \\
Age & $1.04(1.02-1.06)$ & 22.84 & 0.627 \\
Male sex & $0.90(0.58-1.39)$ & 0.24 & 0.133 \\
Hypertension & $1.38(0.90-2.11)$ & 2.25 & 0.793 \\
Diabetes & $1.09(0.57-2.08)$ & 0.07 & 0.891 \\
Prior CAD & $1.07(0.50-2.23)$ & 0.02 & 0.246 \\
Prior CVE & $1.60(0.72-3.57)$ & 1.35 & 0.329 \\
Dyslipidemia & $0.73(0.39-1.37)$ & 0.95 & 0.270 \\
Smoking* & $1.17(0.88-1.55)$ & 1.21 & 0.310 \\
Radiation dose & $1.0(1.0)$ & 1.03 & 0.832 \\
Anti-platelet \& Anti-thrombotic meds ${ }^{\dagger}$ & $0.90(0.36-2.28)$ & 0.05 & \\
\hline
\end{tabular}

$\mathrm{HR}$, hazard ratio; $\mathrm{Cl}$, confidence interval; $\mathrm{CAD}$, coronary artery disease; $\mathrm{CVE}$, cerebrovascular event.

*Smoking: active or prior smoking; ${ }^{\dagger}$ Anti-ischemic meds: Aspirin and/or Plavix and/or Coumadin. 
Supplementary Table 4. Risk of cerebrovascular event following neck radiotherapy using a multivariable Cox regression model for those without advanced disease*

\begin{tabular}{lccr}
\hline Variable & $\mathrm{HR}(95 \% \mathrm{Cl})$ & Chi-square & \multicolumn{1}{c}{$P$} \\
\hline Statin use & $0.40(0.20-0.79)$ & 6.93 & $<.008$ \\
Age & $1.05(1.03-1.07)$ & 25.46 & 0.001 \\
Male sex & $1.09(0.69-1.74)$ & 0.14 & 0.707 \\
Hypertension & $1.26(0.79-1.99)$ & 0.96 & 0.328 \\
Diabetes & $1.01(0.51-2.01)$ & $<0.01$ & 0.981 \\
Prior CAD & $1.26(0.59-2.71)$ & 0.35 & 0.555 \\
Prior CVE & $1.78(0.80-3.94)$ & 1.99 & 0.158 \\
Dyslipidemia & $0.76(0.40-1.45)$ & 0.69 & 0.405 \\
Smoking & $1.32(0.98-1.80)$ & 3.26 & 0.071 \\
\hline
\end{tabular}

$\mathrm{HR}$, hazard ratio; $\mathrm{Cl}$, confidence interval; $\mathrm{CAD}$, coronary artery disease; $\mathrm{CVE}$, cerebrovascular event.

*Advanced disease: stage IV cancer; 'Smoking: active or prior smoking. 